\title{
HILBERT SPACE AND VARIATIONAL METHODS FOR SINGULAR SELFADJOINT SYSTEMS OF DIFFERENTIAL EQUATIONS ${ }^{1}$
}

\author{
BY JUNIOR STEIN ${ }^{2}$
}

Communicated by M. Protter, October 30, 1973

1. Introduction. Let $p, q$, and $r$ be certain $n \times n$ matrix-valued functions with real elements defined almost everywhere on the interval $(a, b)$. Assume $r$ is positive definite a.e. in $[a, b]$. We are going to consider a second order selfadjoint system of ordinary differential equations described by

$$
\left(r(t) \dot{x}(t)+q^{*}(t) x(t)\right)^{\prime}=(q(t) \dot{x}(t)+p(t) x(t)),
$$

where $x$ lies in a certain class of $n$-vector valued functions on $[a, b]$. The differential equation (1.1) is said to be singular at a point $t$ in $[a, b]$ if $r(t)$ is not positive definite. In this paper we restrict our attention to one singularity at $t=a$. However, much of the results carry over if we consider infinitely many singularities to the extent that the Lebesgue measure of the set of singular points is zero.

(1.1) is the Euler equation for the quadratic functional

$$
\begin{aligned}
J(x)= & x^{*}(c) k x(c) \\
& +\int_{a}^{b}\left\{\dot{x}^{*}(t) r(t) \dot{x}(t)+2 x^{*}(t) q(t) \dot{x}(t)+x^{*}(t) p(t) x(t)\right\} d t,
\end{aligned}
$$

where $k$ is an $n \times n$ symmetric matrix with $c$ in $(a, b)$ and $r(c)$ positive definite. Under appropriate conditions, $J$ is well defined on the Hilbert space $A$ of functions $x$ defined on $(a, b]$ and absolutely continuous on each closed subinterval of $(a, b]$ with $\dot{x}^{*} R \dot{x}$ Lebesgue integrable on $(a, b]$. The inner product on $A$ is given by

$$
(x, y)=x^{*}(c) y(c)+\int_{a}^{b} \dot{x}^{*}(t) R(t) \dot{x}(t) d t,
$$

AMS (MOS) subject classifications (1970). Primary 34C05, 34C10; Secondary 46E35. Key words and phrases. Singular differential equations, Hilbert space methods.

${ }^{1}$ The author is indebted to Professor Magnus R. Hestenes for suggesting this problem and for his suggestions in its preparation.

${ }^{2}$ This is to acknowledge the partial support of the author by the U.S. Army Research Office at Durham under Grant DA-31-124-ARO(D)-355 and under Grant DA-AROD-31-124-71-G18. Reproduction in whole or in part is permitted for any purpose of the United States Government. 
where $R$ is symmetric, continuous, and positive definite on $(a, b]$. This Hilbert space is a generalization of the $\alpha$-admissible functions of Morse and Leighton [4, p. 275]. The main idea is to apply theorems comparing the extremals associated with the norm obtained from (1.3) with extremals of $J$ given by (1.2). Since the Hilbert space is constructed so that the norm extremals are known, one is able to obtain information about the extremals of $J$, that is, solutions of (1.1). The singularities considered here need not be regular.

2. Announcement of results. The purpose of this paper is to announce the results that appear in this section. The details and more results are to appear elsewhere. As for terminology and preliminary results, refer to Hestenes [1], [2], [3], Morse and Leighton [4], and Stein [5].

In (1.2) and (1.3), we set $c$ equal to $b$, and assume that $r(t)$ is positive definite for $t$ in $(a, b]$. For $a<e<b, B(e)=\{x \in A: x(t)=0$ if $a<t<e\}, B_{U}$ represents the union of $B(e)$ for $a<e<b, C(e)=\{x \in B(e): x(b)=0\}$, $C_{U}=\left\{x \in B_{U}: x(b)=0\right\}, B$ denotes the closure in $A$ of $B_{U}, C=\{x \in B$ : $x(b)=0\}, D=\{x \in A: x(b)=0\}$, and $A^{+}$possesses the properties of $A$ except that the condition $\dot{x}^{*} R \dot{x}$ Lebesgue integrable on $(a, b]$ is replaced by $\dot{x}^{*} R \dot{x}$ locally integrable on $(a, b]$. By the class of norm extremals we mean $\left\{x \in A^{+}:(x, y)=0\right.$ for all $y$ in $\left.C_{U}\right\}$, and by the class of $J$-extremals we mean $\left\{x \in A^{+}: J(x, y)=0\right.$ for all $y$ in $\left.C_{U}\right\}$. The norm extremals are of the form

$$
x(t)=\left(\int_{b}^{t} R^{-1}(s) d s\right) c_{0}+x(b) .
$$

Let

$$
Z(t)=I+\int_{t}^{b} R^{-1}(s) d s,
$$

where $I$ is the $n \times n$ identity matrix. A norm extremal (2.1) is in $A$ if and only if $c_{0}^{*} Z(a) c_{0}<\infty$. Accordingly, we say that our norm is extremally complete if $Z(t)$ is bounded on $(a, b]$. Let $\lambda_{1}(t) \leqq \lambda_{2}(t) \leqq \cdots \leqq \lambda_{n}(t)$ denote the ordered eigenvalues of $Z(t)$ for all $t$ in $(a, b]$. We say that the norm is extremally incomplete of degree $k(1 \leqq k \leqq n)$ if

$$
\begin{array}{ll}
\lim _{t \rightarrow a+} \lambda_{i}(t)=+\infty & (n-k+1 \leqq i \leqq n), \\
\lim _{t \rightarrow a+} \lambda_{i}(t)<+\infty & (1 \leqq i \leqq n-k) .
\end{array}
$$

We say that extremal completeness is extremal incompleteness of degree zero and that the norm is totally extremally incomplete if it is incompete of degree $n$. 
THeOREM 2.1. Assume that both $p$ and $q$ are measurable $n \times n$ matrixvalued functions on $(a, b)$. Further assume that $p$ is symmetric almost everywhere on $(a, b)$. Let

$$
P=Z^{1 / 2} p Z^{1 / 2} \text { and } Q=Z^{1 / 2} q R^{-1} q^{*} Z^{1 / 2}
$$

be Lebesgue integrable on $(a, b)$, where $Z$ is given by (2.2). Let $k$ be any constant $n \times n$ matrix. Then

$$
K(x)=x^{*}(b) k x(b)+\int_{a}^{b}\left\{2 x^{*}(t) q(t) \dot{x}(t)+x^{*}(t) p(t) x(t)\right\} d t
$$

has the property that $K\left(x_{n}\right) \rightarrow 0$ whenever $x_{n} \rightarrow 0$ weakly in $A$, and hence $K$ is bounded on $A$.

THeOREM 2.2. Assume that $P=Z^{1 / 2} p Z^{1 / 2}$ and $Q=Z^{1 / 2} q R^{-1} q^{*} Z^{1 / 2}$ are Lebesgue integrable on $(a, b)$ where $Z$ is given by (2.2). Let $p, q$, and $r$ be measurable $n \times n$ matrix-valued functions on $(a, b)$ with $p$ and $r$ symmetric. Let $k$ be any constant $n \times n$ matrix. Let

where

$$
J(x)=\int_{a}^{b}\left\{\dot{x}^{*}(t) r(t) \dot{x}(t)\right\} d t+K(x)
$$

$$
K(x)=x^{*}(b) k x(b)+\int_{a}^{b}\left\{2 x^{*}(t) q(t) \dot{x}(t)+x^{*}(t) p(t) x(t)\right\} d t .
$$

If $r$ is locally integrable on $(a, b)$ and $J$ is well defined on the Hilbert space $A$, then

(i) $J$ is weakly lower semicontinuous on $A$ if and only if $r \geqq 0$ almost everywhere on $(a, b)$.

(ii) $J$ is bounded on $A$ if and only if there is a positive constant $c$ such that for each vector $v$ in Euclidean $n$-space $\left|v^{*} r(t) v\right| \leqq c v^{*} R(t) v$ for almost all $t$ in $(a, b)$.

(iii) $J$ is compact on $A$ if and only if $r=0$ almost everywhere on $(a, b)$.

(iv) $J$ is positively elliptic on $A$ if and only if there are positive numbers $c_{1}$ and $c_{2}$ such that $c_{1} R(t) \leqq r(t) \leqq c_{2} R(t)$ for almost all $t$ in $(a, b)$.

THEOREM 2.3. The degree of norm extremal incompleteness is $k$ if and only if the dimension of the class of norm extremals in $A$ is $2 n-k$ where $0 \leqq k \leqq n$.

LEMMA 2.1. Let $Q$ be a quasi-nonsingular bilinear form defined on a Hilbert space $H$ with the nullity of $Q$ on $H$ finite. Let $S$ be a closed subspace of $H$ with $\operatorname{dim} S^{\perp}$ finite. Let $S^{\perp}=S^{\prime}+S^{\prime \prime}$ where

Then

$$
S^{\prime}=S^{\perp} \cap\left(H^{Q}\right)^{\perp} \text { and } S^{\prime \prime}=S^{\perp} \cap\left(S^{\prime}\right)^{\perp} .
$$

$$
\operatorname{dim} S^{\prime \prime}=\operatorname{dim} H^{Q}-\operatorname{dim}\left(S \cap H^{Q}\right) .
$$


THEOREM 2.4. Let $Q$ be a quasi-nonsingular bilinear form defined on a Hilbert space $H$ with the nullity of $Q$ on $H$ finite. Let $S$ be a closed subspace of $H$ with $\operatorname{dim} S^{\perp}$ finite. Then we have

$$
\operatorname{dim} S^{Q}=\operatorname{dim} S^{\perp}+\operatorname{dim}\left(S \cap H^{Q}\right) .
$$

Corollary 2.1. Assume that $J$ is a quasi-nonsingular form on $A$ with $\operatorname{dim} A^{J}<\infty$ and $\operatorname{det} r(b) \neq 0$. The dimension of the class of J-extremals in $A$ equals the dimension of the class of norm extremals in $A$.

Corollary 2.2. Assume that $J$ is a quasi-nonsingular form on $A$ with $\operatorname{dim} A^{J}<\infty$ and $\operatorname{det} r(b) \neq 0$. If the norm is extremally complete, then all the J-extremals are in $A$. If the norm is totally extremally incomplete, then there are exactly $n$ linearly independent J-extremals in $A$. If the norm is extremally incomplete of degree $k$ where $0 \leqq k \leqq n$, then there are precisely $2 n-k$ linearly independent $J$-extremals in $A$.

THEOREM 2.5. Let $k$ be the degree of norm extremal incompleteness where $0 \leqq k \leqq n$. Then there is an $n \times(n-k)$ matrix $F$ of rank $n-k$ such that $B=\left\{x \in A: x^{*}(a) F=0\right\}, C=\left\{x \in A: x^{*}(a) F=0\right.$ and $\left.x(b)=0\right\}$.

Legendre's differential equation has a singularity at $t=-1$. By taking $R(t)=1-t$ where $r(t)=(1-t)(1+t)$ for $-1<t \leqq 0$, we have an example of total extremal incompleteness. From this we can conclude that there is a solution bounded on $(-1,0]$ and another unbounded there. Chebyshev's differential equation provides us with an example of norm extremal completeness, and every solution is bounded on $(-1,1)$.

\section{BIBLIOGRAPHY}

1. M. R. Hestenes, Applications of the theory of quadratic forms in Hilbert space to the calculus of variations, Pacific J. Math. 1 (1951), 525-581. MR 13, 759.

2. —-, Quadratic control problems, Class Notes, Math. Dept. Reading Room, University of California, Los Angeles, Calif., 1969.

3. - Quadratic variational theory, Control Theory and the Calculus of Variations (A. V. Balakrishnan, editor), Academic Press, New York, 1969, pp. 1-37.

4. M. Morse and W. Leighton, Singular quadratic functionals, Trans. Amer. Math. Soc. 40 (1936), 252-286.

5. J. Stein, Singular quadratic functionals, Dissertation, University of California, Los Angeles, Calif., 1971.

Department of Mathematics, University of Toledo, Toledo, Ohio 43606 